\title{
A Multiple-Comparison-Systems Method for Distributed Stability Analysis of Large-Scale Nonlinear Systems
}

\author{
Soumya Kundu ${ }^{\text {a }}$, Marian Anghel ${ }^{\mathrm{b}}$ \\ ${ }^{a}$ Advanced Controls Group, Pacific Northwest National Laboratory, Richland, WA 99354, USA \\ b Information Sciences Group, Los Alamos National Laboratory, Los Alamos, NM 87545, USA
}

\begin{abstract}
Lyapunov functions provide a tool to analyze the stability of nonlinear systems without extensively solving the dynamics. Recent advances in sum-of-squares methods have enabled the algorithmic computation of Lyapunov functions for polynomial systems. However, for general large-scale nonlinear networks it is yet very difficult, and often impossible, both computationally and analytically, to find Lyapunov functions. In such cases, a system decomposition coupled to a vector Lyapunov functions approach provides a feasible alternative by analyzing the stability of the nonlinear network through a reduced-order comparison system. However, finding such a comparison system is not trivial and often, for a nonlinear network, there does not exist a single comparison system. In this work, we propose a multiple comparison systems approach for the algorithmic stability analysis of nonlinear systems. Using sum-of-squares methods we design a scalable and distributed algorithm which enables the computation of comparison systems using only communications between the neighboring subsystems. We demonstrate the algorithm by applying it to an arbitrarily generated network of interacting Van der Pol oscillators.
\end{abstract}

Key words: Lyapunov stability, dynamical systems, sum-of-squares optimization, disturbance analysis, interconnected systems.

\section{Introduction}

A key to maintaining the successful operation of real-world engineering systems is to analyze the stability of the systems under disturbances. Lyapunov functions methods provide powerful tools to directly certify stability under disturbances, without solving the complex nonlinear dynamical equations $[17,26]$. However for a general nonlinear system, there is no universal expression for Lyapunov functions. Recent advances in sum-of-squares (SOS) methods and semi-definite programming (SDP), [29,32,34], have enabled the algorithmic construction of polynomial Lyapunov functions for nonlinear systems that can be expressed as a set of polynomial differential algebraic equations $[12,36]$. Unfortunately, such computational methods suffer from scalability issues and, in general, become intractable as the system size grows [2]. For this reason more tractable alternatives to SOS optimization have been proposed. One such approach, known as DSOS and SDSOS optimization, is significantly more scalable since it relies on linear program-

\footnotetext{
* The work was performed when Soumya Kundu was with Center for Nonlinear Studies at Los Alamos National Laboratory. The authors are grateful to the U.S. Department of Energy for supporting this research work through LANL/LDRD program.

Email addresses: soumya . kundu@pnnl .gov (Soumya Kundu), manghelelanl.gov (Marian Anghel).
}

ming and second order cone programming [1]. A different approach chooses Lyapunov functions with a chordal graphical structure in order to convert the semidefinite constraints into an equivalent set of smaller semidefinite constraints which can be exploited to solve the SDP programs more efficiently [27]. Nevertheless, the increased scalability decreases performance since both approximations are usually more conservative than SOS approaches.

Despite these computational advances, global analysis of large-scale systems remains problematic when computational and communication costs are considered. Often, a decomposition-aggregation approach offers a scalable distributed computing framework, together with a flexible analysis of structural perturbations [14] and decentralized control designs [40], as required by the locality of perturbations. Thus, for large-scale systems, it is often useful to model the system as a network of small interacting subsystems and study the stability of the full interconnected system with the help of the Lyapunov functions of the isolated subsystems. For example, one approach is to construct a scalar Lyapunov function expressed as a weighted sum of the subsystem Lyapunov functions and use it to certify stability of the full system $[4,28,39,41]$. However, such a method requires centralized computations and does not scale well with the size of the network. Alternatively, methods based on vector Lyapunov functions, [5,8], are computationally 
very attractive due to their parallel structure and scalability, and have generated considerable interest in recent times [20, 23, 24, 43]. However, applicability of these methods to large-scale nonlinear systems with guaranteed rate of convergence still remains to be explored. For example, in $[23,24]$ authors consider asymptotic stability while the works in $[20,43]$ are demonstrated on small-scale systems.

Inspired by the results on comparison systems, $[6,9,13]$, it has been observed that the problem of stability analysis of an interconnected nonlinear system can be reduced to the stability analysis of a linear dynamical system (or, 'single comparison system') whose state space consists of the subsystem Lyapunov functions. Success in finding such stable linear comparison system then guarantees exponential stability of the full interconnected nonlinear system. However, for a given interconnected system, computing these comparison systems still remained a challenge. In absence of suitable computational tools, analytical insights were used to build those comparison systems, such as trigonometric inequalities in power systems networks [19]. In a recent work [22], SOS-based direct methods were used to compute the single comparison system for generic nonlinear polynomial systems, with some performance improvements over the traditional methods. However there are major challenges before such a method can be used in large-scale systems. For example, it is generally difficult to construct a single comparison system that can guarantee stability under a wide set of disturbances. Also, while [22] presents a decentralized analysis where the computational burden is shared between the subsystems, the scalability of the analysis is largely dependent on the cumulative size of the neighboring subsystems.

In this article we present a novel conceptual and computational framework which generalizes the single comparison system approach into a sequence of stable comparison systems, that collectively ascertain stability, while also offering better scalability by parallelizing the subsystem-level SOS problems. The set of multiple comparison systems are to be constructed adaptively in real-time, after a disturbance has occurred. With the help of SOS and semi-definite programming methods, we develop a fully distributed, parallel and scalable algorithm that enables computation of the comparison systems under a disturbance, with only minimal communication between the immediate neighbors. While this approach is applicable to any generic dynamical system, we choose an arbitrarily generated network of modified ${ }^{1}$ Van der Pol oscillators [38] for illustration. Under a disturbance, the subsystems communicate with their neighbors to algorithmically construct a set of multiple comparison systems, the successful construction of which can certify stability of the network. The rest of this article is organized as follows. Following some brief background in Section 2 we describe the problem in Section 3. We present the traditional approach to single comparison systems and an SOS-based direct method of computing the comparison systems in Section 4. In Section 5, we introduce the concept of multiple

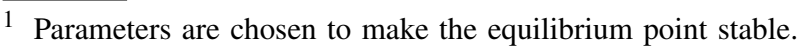

comparison systems, and propose a parallel and distributed algorithmic construction of the comparison systems in realtime. We demonstrate an application of this algorithm to a network of Van der Pol oscillators in Section 6, before concluding the article in Section 7.

\section{Preliminaries}

Let us consider the dynamical system

$$
\dot{x}(t)=f(x(t)), \quad t \geq 0, x \in \mathbb{R}^{n}, f(0)=0,
$$

with an equilibrium at the origin ${ }^{2}$, and $f: \mathbb{R}^{n} \rightarrow \mathbb{R}^{n}$ is locally Lipschitz. Let us use $|\cdot|$ to denote both the Euclidean norm (for a vector) and the absolute value (for a scalar).

Definition 1 The equilibrium point at the origin is said to be asymptotically stable in a domain $\mathscr{D} \subseteq \mathbb{R}^{n}, 0 \in \mathscr{D}$, if $\lim _{t \rightarrow \infty}|x(t)|=0$ for every $|x(0)| \in \mathscr{D}$, and it is exponentially stable if there exists $b, c>0$ such that $|x(t)|<$ $c e^{-b t}|x(0)| \forall t \geq 0$, for every $|x(0)| \in \mathscr{D}$.

Theorem 1 (Lyapunov, [26], [21], Thm. 4.1) If there exists a domain $\mathscr{D} \subseteq \mathbb{R}^{n}, 0 \in \mathscr{D}$, and a continuously differentiable positive definite function $\tilde{V}: \mathscr{D} \rightarrow \mathbb{R}_{\geq 0}$, i.e. the 'Lyapunov function' ( $L F)$, then the origin of (1) is asymptotically stable if $\nabla \tilde{V}^{T} f(x)$ is negative definite in $\mathscr{D}$, and is exponentially stable if $\nabla \tilde{V}^{T} f(x) \leq-\alpha \tilde{V} \forall x \in \mathscr{D}$, for some $\alpha>0$.

The region of attraction (ROA) of the stable equilibrium point at origin can be (conservatively) estimated as [15]

$$
\begin{gathered}
\mathscr{R}:=\{x \in \mathscr{D} \mid V(x) \leq 1\} \text {, with } V(x)=\tilde{V}(x) / \gamma^{\max }, \\
\text { where } \gamma^{\max }:=\max \left\{\gamma \mid\left\{x \in \mathbb{R}^{n} \mid \tilde{V}(x) \leq \gamma\right\} \subseteq \mathscr{D}\right\},
\end{gathered}
$$

i.e. the boundary of the ROA is estimated by the unit levelset of a suitably scaled LF $V(x)$. Relatively recent studies have explored how sum-of-squares (SOS) based methods can be utilized to find LFs by restricting the search space to SOS polynomials $[3,18,31,35]$. Let us denote by $\mathbb{R}[x]$ the ring of all polynomials in $x \in \mathbb{R}^{n}$.

Definition 2 A multivariate polynomial $p \in \mathbb{R}[x], x \in \mathbb{R}^{n}$, is a sum-of-squares (SOS) if there exist some polynomial functions $h_{i}(x), i=1 \ldots s$ such that $p(x)=\sum_{i=1}^{s} h_{i}^{2}(x)$. We denote the ring of all SOS polynomials in $x \in \mathbb{R}^{n}$ by $\Sigma[x]$.

Checking if $p \in \mathbb{R}[x]$ is an SOS is a semi-definite problem which can be solved with a MATLAB ${ }^{\circledR}$ toolbox SOSTOOLS $[29,32]$ along with a semidefinite programming solver such as SeDuMi [34]. The SOS technique can be used to search for polynomial LFs by translating the conditions

2 Note that by shifting the state variables any equilibrium point of interest can be moved to the origin. 
in Theorem 1 to equivalent SOS conditions $[11,18,29,30$, $32,42]$. An important result from algebraic geometry, called Putinar's Positivstellensatz theorem [25,33], helps in translating the SOS conditions into SOS feasibility problems. The Putinar's Positivestellensatz theorem states (see [25], Ch. 2)

Theorem 2 Let $\mathscr{K}=\left\{x \in \mathbb{R}^{n} \mid k_{1}(x) \geq 0, \ldots, k_{m}(x) \geq 0\right\}$ be a compact set, where $k_{j} \in \mathbb{R}[x], \forall j \in\{1, \ldots, m\}$. Suppose there exists a $\mu \in\left\{\sigma_{0}+\sum_{j=1}^{m} \sigma_{j} k_{j} \mid \sigma_{0}, \sigma_{j} \in \Sigma[x], \forall j\right\}$ such that $\left\{x \in \mathbb{R}^{n} \mid \mu(x) \geq 0\right\}$ is compact. Then, if $p(x)>0 \forall x \in$ $\mathscr{K}$, then $p \in\left\{\sigma_{0}+\sum_{j} \sigma_{j} k_{j} \mid \sigma_{0}, \sigma_{j} \in \Sigma[x], \forall j\right\}$.

Remark 1 Using Theorem 2, we can translate the problem of checking that $p>0$ on $\mathscr{K}$ into an SOS feasibility problem where we seek the SOS polynomials $\sigma_{0}, \sigma_{j} \forall j$ such that $p-\sum_{j} \sigma_{j} k_{j}$ is SOS. Note that any equality constraint $k_{i}(x)=0$ can be expressed as two inequalities $k_{i}(x) \geq 0$ and $k_{i}(x) \leq 0$. In many cases, especially for the $k_{i} \forall i$ used throughout this work, a $\mu$ satisfying the conditions in Theorem 2 is guaranteed to exist (see [25]), and need not be searched for.

In $[9,13]$ the authors proposed to view the LF as a dependent variable in a first-order auxiliary differential equation, often termed as the 'comparison equation' (or, 'comparison system'). It was shown in $[5,8]$ that, under certain conditions, the comparison equation can be effectively reduced to a set of linear differential equations. Noting that all the elements of the matrix $e^{A t}, t \geq 0$, where $A=\left[a_{i j}\right] \in \mathbb{R}^{m \times m}$, are nonnegative if and only if $a_{i j} \geq 0, i \neq j$, it was shown in [6,8]:

Lemma 1 Let $A \in \mathbb{R}^{m \times m}$ have non-negative off-diagonal elements, $v:[0, \infty) \rightarrow \mathbb{R}^{m}$ and $r:[0, \infty) \rightarrow \mathbb{R}^{m}$. If $v(0)=r(0)$, $\dot{v}(t) \leq A v(t)$ and $\dot{r}(t)=A r(t)$, then $v(t) \leq r(t) \forall t \geq 0$.

We henceforth refer to Lemma 1 as the "comparison principle' and the differential inequalities of the form $\dot{v}(t) \leq A v(t)$ as a 'comparison system' (CS).

\section{Problem Description}

Let us consider a network of $m$ (locally) asymptotically stable, polynomial ${ }^{3}$ subsystems represented as follows,

$$
\begin{aligned}
\forall i \in\{1, \ldots, m\}: & \dot{x}_{i}=f_{i}\left(x_{i}\right)+g_{i}(x), x_{i} \in \mathbb{R}^{n_{i}}, x \in \mathbb{R}^{n} \\
& g_{i}(x)=\sum_{j \neq i} g_{i j}\left(x_{i}, x_{j}\right), \\
& f_{i}(0)=0, g_{i j}\left(x_{i}, 0\right)=0 \forall j \neq i,
\end{aligned}
$$

where $x_{i}$ are the state variables that belong to the $i^{\text {th }}$ subsystem, $f_{i} \in \mathbb{R}\left[x_{i}\right]^{n_{i}}$ denotes the isolated subsystem dynamics,

\footnotetext{
3 We consider the cases when a non-polynomial dynamics can be recasted into an equivalent (exact) polynomial form, with additional variables and constraints $[3,30]$. Otherwise, we can use Taylor expansions for the non-polynomial terms and a careful analysis of the worst case remainders as in [10].
}

$g_{i} \in \mathbb{R}[x]^{n_{i}}$ represents the total neighbor interactions. We assume, for simplicity, that the total interaction term $g_{i}$ can be split into a sum of pairwise interactions, $g_{i j} \in \mathbb{R}\left[x_{i}, x_{j}\right]^{n_{i}}$. When this assumption is invalid, the algorithm proposed is still applicable, with either a re-definition of the subsystems or a slight modification of the formulation (esp. Section 5.2). Note that subsystems may share common state variable(s), i.e. $n \leq \sum_{j=1}^{m} n_{j}$. Finally,

$$
\begin{aligned}
\mathscr{N}_{i} & :=\{i\} \bigcup\left\{j \mid \exists\left\{x_{i}, x_{j}\right\}, \text { s.t. } g_{i j}\left(x_{i}, x_{j}\right) \neq 0\right\}, \\
\text { and } \bar{x}_{i} & :=\bigcup_{j \in \mathscr{N}_{i}}\left\{x_{j}\right\},
\end{aligned}
$$

denote the set of the subsystems in the neighborhood of the $i^{\text {th }}$ subsystem (including itself) and the state variables that belong to this neighborhood, respectively.

The polynomial LFs, $V_{i} \in \mathbb{R}\left[x_{i}\right] \forall i$, for the isolated subsystems, $\dot{x}_{i}=f_{i}\left(x_{i}\right), \forall i$, are computed using an SOS-based $e x$ panding interior algorithm [3,18] (alternatively, the methods in $[12,37]$ can be used), with the isolated ROAs

$$
\mathscr{R}_{i}^{0}:=\left\{x_{i} \in \mathbb{R}^{n_{i}} \mid V_{i}\left(x_{i}\right) \leq 1\right\}, \forall i \in\{1,2, \ldots, m\} .
$$

The $V_{i} \forall i$ satisfy, for some $\eta_{i 1}, \eta_{i 2}, \eta_{i 3}>0 \forall i$ and $\mathscr{D}_{i} \subset \mathscr{R}_{i}^{0} \forall i$,

$$
\begin{gathered}
\forall i: \forall x_{i} \in \mathscr{D}_{i} \subset \mathscr{R}_{i}^{0}, \eta_{i 1}\left|x_{i}\right|^{d_{i}} \leq V_{i}\left(x_{i}\right) \leq \eta_{i 2}\left|x_{i}\right|^{d_{i}}, \\
\text { and } \nabla V_{i}^{T} f_{i} \leq-\eta_{i 3}\left|x_{i}\right|^{d_{i}},
\end{gathered}
$$

where, $d_{i}$ is an even positive integer denoting the lowest degree among all monomials in $V_{i}\left(x_{i}\right)$. Further, there exist $\zeta_{i j}>0 \forall i, \forall j \in \mathscr{N}_{i} \backslash\{i\}$, such that the interactions $g_{i j}$ satisfy:

$$
\forall x_{i} \in \mathscr{D}_{i}, \forall x_{j} \in \mathscr{D}_{j},\left|\nabla V_{i}^{T} g_{i j}\right| \leq \zeta_{i j}\left|x_{i}\right|^{d_{i}-1}\left|x_{j}\right| .
$$

Let us introduce the 'self-decay rates', $\alpha_{i}:(0,1] \rightarrow \mathbb{R}_{\geq 0}$, $\alpha_{i}(1)=0$, of the subsystem LFs, $\forall \gamma_{i} \in(0,1]$, as

$$
\alpha_{i}\left(\gamma_{i}\right):=\max \left\{\alpha \geq 0 \mid \nabla V_{i}^{T} f_{i} \leq-\alpha V_{i} \text { on } V_{i}\left(x_{i}\right)=\gamma_{i}\right\} .
$$

We will address two stability problems in this paper. First, assume that a disturbance is applied to the link between subsystems $i$ and $j$ (a fault in power systems). This means that $g_{i j}\left(x_{i}, x_{j}\right)$ in (3b) changes, or even becomes 0 if the link is cut, and the system moves away from its equilibrium point. After the fault is cleared, we reset the clock to 0 , and consider the evolution of system (3) from the state $x(0) \neq 0$. Thus, any disturbance moves the system away from the equilibrium and results in positive level-sets $V_{i}\left(x_{i}(0)\right)=v_{i}^{0} \in(0,1]$ for some or all of the subsystems. A stability problem can be then formulated as checking if $\lim _{t \rightarrow+\infty} V_{i}\left(x_{i}(t)\right)=0 \forall i$ whenever $V_{i}\left(x_{i}(0)\right)=v_{i}^{0} \forall i$, where $x_{i}(t), t>0$, are solutions of the coupled dynamics in (3). An attractive and scalable approach to solving this problem is to construct a vector LF $V: \mathbb{R}^{n} \rightarrow \mathbb{R}_{\geq 0}^{m}$, defined as:

$$
V(x):=\left[\begin{array}{llll}
V_{1}\left(x_{1}\right) & V_{2}\left(x_{2}\right) & \ldots & V_{m}\left(x_{m}\right)
\end{array}\right]^{T},
$$


and use a 'comparison system' to certify that $\lim _{t \rightarrow+\infty} V(x(t))=$ 0 . Restricting our focus to the linear comparison principle (Lemma 1), the aim is to seek an $A=\left[a_{i j}\right] \in \mathbb{R}^{m \times m}$ and a domain $\mathscr{R} \subseteq\left\{x \in \mathbb{R}^{n} \mid x_{i} \in \mathscr{R}_{i}^{0} \forall i\right\}$, with $0 \in \mathscr{R}$, such that

$$
\dot{V}(x)=\nabla V^{T} f(x) \leq A V(x), \forall x \in \mathscr{R},
$$

where, $A=\left[a_{i j}\right]$ is Hurwitz, $a_{i j}>0 \forall i \neq j$,

and $\mathscr{R}$ is invariant under the dynamics (1).

Henceforth, we refer to a comparison system of the form (10a) as a 'single comparison system', since one matrix $A=\left[a_{i j}\right]$ satisfies the differential inequalities in the full domain $\mathscr{R}$ which includes the origin. When (10) holds ${ }^{4}$, any $x(0) \in \mathscr{R}$ would imply exponential convergence of $V(x(t))$ to the origin (from Lemma 1), which, via (6a), also implies exponential convergence of the states [39].

A second stability problem is to seek an optimal estimate of the region of attraction (ROA) of the stable equilibrium point by maximizing the domain $\mathscr{R}$ in (10). While such optimization problems are difficult, and are not the main scope of this paper, we will describe in Section 6 the results of estimating the ROA for a particular optimization direction.

\section{Single Comparison System}

Let us first review the traditional approach towards stability analysis of interconnected dynamical systems using a single comparison system (or, single CS). In [4, 19, 39, 41], and related works, authors laid out a formulation of the linear CS using certain conditions on the LFs and the neighbor interactions. It was observed that if there exists a set of LFs, $v_{i}: \mathbb{R}^{n_{i}} \rightarrow \mathbb{R}_{\geq 0}, \forall i=1,2, \ldots, m$, satisfying the following

$$
\begin{array}{r}
\forall(i, j): \quad \tilde{\eta}_{i 1}\left|x_{i}\right| \leq v_{i}\left(x_{i}\right) \leq \tilde{\eta}_{i 2}\left|x_{i}\right|, \forall x_{i} \in \tilde{\mathscr{D}}_{i} \subset \mathscr{R}_{i}^{0}, \\
\left(\nabla v_{i}\right)^{T} f_{i} \leq-\tilde{\eta}_{i 3}\left|x_{i}\right|, \forall x_{i} \in \tilde{\mathscr{D}}_{i} \subset \mathscr{R}_{i}^{0}, \\
\text { and }\left|\left(\nabla v_{i}\right)^{T} g_{i j}\right| \leq \tilde{\zeta}_{i j}\left|x_{j}\right|, \forall x_{i} \in \tilde{\mathscr{D}}_{i}, \forall x_{j} \in \tilde{\mathscr{D}}_{j}
\end{array}
$$

for some $\tilde{\eta}_{i 1}, \tilde{\eta}_{i 2}, \tilde{\eta}_{i 3}>0 \forall i$ and $\tilde{\zeta}_{i j} \geq 0 \forall(i, j)$, with $\tilde{\zeta}_{i j}=$ $0 \forall j \notin \mathscr{N}_{i}$, then the corresponding vector LF $v=\left[\begin{array}{lll}v_{1} & v_{2} \ldots v_{m}\end{array}\right]^{T}$ satisfies a CS on the domain $\tilde{\mathscr{D}}=\left\{x \in \mathbb{R}^{n} \mid x_{i} \in \tilde{\mathscr{D}}_{i} \forall i\right\}$, with a comparison matrix $\tilde{A}=\left[\tilde{a}_{i j}\right]$ given by

$$
\forall(i, j): \quad \tilde{a}_{i i}=-\tilde{\eta}_{i 3} / \tilde{\eta}_{i 2}, \tilde{a}_{i j}=\tilde{\zeta}_{i j} / \tilde{\eta}_{j 1}
$$

If $\tilde{A}$ is Hurwitz, then any invariant domain $\mathscr{R} \subseteq \tilde{\mathscr{D}}$ is an estimate of a region of exponential stability $[19,41]$.

Although the traditional approach provides nice analytical insights into the construction of the comparison matrix, it suffers from practical limitations. One has to first compute

$4 A=\left[a_{i j}\right]$ is called Hurwitz if its eigenvalues have negative real parts. $\mathscr{R}$ is called invariant if $x(0) \in \mathscr{R} \Longrightarrow x(t) \in \mathscr{R} \forall t>0$. the bounds in (11) which, interestingly, are not satisfied by any polynomial LFs. However, one can compute the bounds in (6) satisfied by the polynomial LFs, and then construct a set of non-polynomial LFs as $[19,41]$

$$
\begin{gathered}
\forall(i, j): v_{i}=\sqrt[d_{i}]{V_{i}} \text { satisfies (11) with } \\
\tilde{\eta}_{i 1}=\sqrt[d_{i}]{\eta_{i 1}}, \quad \tilde{\eta}_{i 2}=\sqrt[d_{i}]{\eta_{i 2}}, \quad \tilde{\eta}_{i 3}=\frac{\eta_{i 3} \tilde{\eta}_{i 2}}{d_{i} \eta_{i 2}}, \tilde{\zeta}_{i j}=\frac{\zeta_{i j} \tilde{\eta}_{i 1}}{d_{i} \eta_{i 1}}
\end{gathered}
$$

Further, the bounds in (11), while convenient for analytical insights, need not be optimal for constructing a Hurwitz comparison matrix. For example, in (11c), $\left|\nabla v_{i}^{T} g_{i j}\right|$ is a function of both $x_{i}$ and $x_{j}$ but is bounded using only $\left|x_{j}\right|$.

\subsection{SOS-Based Direct Computation}

SOS-based techniques can be used to resolve some of the issues that arise with the traditional approach [22]. The idea is to compute the single $C S$ in a decentralized way, using directly the LFs $V_{i} \in \mathbb{R}\left[x_{i}\right][3,18]$, which however do not satisfy the conditions in (11). For convenience, let us introduce, for all $i \in\{1, \ldots, m\}$, the following notations,

$$
\begin{array}{r}
\forall 0 \leq a_{2}<a_{1} \leq 1: \mathscr{D}_{i}\left[a_{1}\right]:=\left\{x \in \mathbb{R}^{n} \mid V_{i}\left(x_{i}\right) \leq a_{1}\right\}, \\
\mathscr{D}_{i}^{b}\left[a_{1}\right]:=\left\{x \in \mathbb{R}^{n} \mid V_{i}\left(x_{i}\right)=a_{1}\right\}, \\
\text { and } \mathscr{D}_{i}\left[a_{1}, a_{2}\right]:=\left\{x \in \mathbb{R}^{n} \mid a_{2}<V_{i}\left(x_{i}\right) \leq a_{1}\right\}
\end{array}
$$

Given a set of $\gamma_{i}^{0} \in(0,1] \forall i$, we want to construct the single $\mathrm{CS}$ in a distributed way by calculating each row of the comparison matrix $A \in \mathbb{R}^{m \times m}$ (with non-negative off-diagonal entries) locally at each subsystem level, such that,

$$
\forall i \in\{1, \ldots, m\}: \dot{V}_{i} \leq \sum_{j \in \mathscr{N}_{i}} a_{i j} V_{j} \text { on } \bigcap_{j \in \mathscr{N}_{i}} \mathscr{D}_{j}\left[\gamma_{j}^{0}\right]
$$

Proposition 1 The domain $\bigcap_{i=1}^{m} \mathscr{D}_{i}\left[\gamma_{i}^{0}\right]$ is an estimate of the ROA of the interconnected system in (3) if for each $i \in$ $\{1,2, \ldots, m\}, \sum_{j \in \mathscr{N}_{i}} a_{i j}<0$ and $\sum_{j \in \mathscr{N}_{i}} a_{i j} \gamma_{j}^{0}<0$.

Proof Because of the non-negative off-diagonal entries and $\sum_{j \in \mathscr{N}_{i}} a_{i j}<0 \forall i$, the application of Gershgorin's Circle theorem $[7,16]$ states that the comparison matrix $A=\left[a_{i j}\right]$ is Hurwitz. Further, note that whenever $V_{i}\left(x_{i}(\tau)\right)=\gamma_{i}^{0}$, for some $i$, and $V_{k}\left(x_{k}(\tau)\right) \leq \gamma_{k}^{0} \forall k \neq i$, for some $\tau \geq 0$, we have $\left.\dot{V}_{i}\left(x_{i}\right)\right|_{t=\tau}<0$. i.e. the (piecewise continuous) trajectories can never cross the boundaries defined by $\bigcap_{i=1}^{m} \mathscr{D}_{i}^{b}\left[\gamma_{i}^{0}\right]$.

Remark 2 Henceforth, we will loosely refer to the conditions of the form $\sum_{j \in N_{i}} a_{i j}<0$ as the 'Hurwitz conditions', while the conditions of the form $\sum_{j \in \mathscr{N}_{i}} a_{i j} \gamma_{j}^{0}<0$ will be referred to as the 'invariance conditions'.

Proposition 1 helps us formulate local (subsystem-level) SOS problems to find the single CS and check if the domain $\bigcap_{i=1}^{m} \mathscr{D}_{i}\left[\gamma_{i}^{0}\right]$ is an estimated ROA. Note that in this 
formulation we use the polynomial LFs that do not satisfy the bounds in (11). However, we prefer to directly use the polynomial LFs, instead of converting them to their non-polynomial counterparts as in (13), for two reasons: 1) convenience of applying SOS methods, and 2) better stability certificates, as shown in the following result.

Proposition 2 If for some LFs $v_{i}, i \in\{1, \ldots, m\}$, there exists a comparison matrix $\tilde{A}=\left[\tilde{a}_{i j}\right]$, with $\tilde{a}_{i i}+\sum_{j \neq i} \tilde{a}_{i j} \tilde{c}_{i j}<0 \forall i \in$ $\{1, \ldots, m\}$, for some $\tilde{c}_{i j}>0 \forall i \neq j$, then, for any LFs $V_{i}=$ $\left(v_{i}\right)^{d} \forall i, d \geq 1$, the existence of a comparison matrix $A=\left[a_{i j}\right]$ is guaranteed, with $a_{i i}+\sum_{j \neq i} a_{i j}\left(\tilde{c}_{i j}\right)^{d}<0 \forall i$.

Proof Note from Proposition 1, that choosing $\tilde{c}_{i j}=1, \gamma_{j}^{0} / \gamma_{i}^{0}$, or $\max \left(\gamma_{j}^{0} / \gamma_{i}^{0}, 1\right)$ we may retrieve the Hurwitz condition, the invariance condition, or simultaneously both, respectively. The proof follows directly after we show that $\forall i \in\{1, \ldots, m\}$,

$$
\begin{aligned}
\dot{V}_{i} & \leq d v_{i}^{d-1} \sum_{j=1}^{m} \tilde{a}_{i j} v_{j}=d \tilde{a}_{i i} V_{i}+d \sum_{j \neq i} \tilde{a}_{i j} \tilde{c}_{i j} V_{i}^{\frac{d-1}{d}}\left(\tilde{c}_{i j}^{-d} V_{j}\right)^{1 / d} \\
& \leq d \tilde{a}_{i i} V_{i}+\sum_{j \neq i} \tilde{a}_{i j} \tilde{c}_{i j}\left((d-1) V_{i}+\tilde{c}_{i j}^{-d} V_{j}\right)=\sum_{j} a_{i j} V_{j}
\end{aligned}
$$

by using Young's inequality ${ }^{5}$, and choosing $a_{i i}=d \tilde{a}_{i i}+(d-$ 1) $\sum_{j \neq i} \tilde{a}_{i j} \tilde{c}_{i j}, \forall i$, and $a_{i j}=\tilde{a}_{i j}\left(\tilde{c}_{i j}\right)^{1-d} \forall i \neq j$.

Motivated by Propositions 1-2, we propose an SOS-based direct computation of the comparison matrix $A=\left[a_{i j}\right]$ in (15), in which each subsystem calculates the corresponding row entries of the matrix $A$, by solving the following SOS feasibility problem (using Theorem 2 ):

$$
\begin{array}{r}
-\nabla V_{i}^{T}\left(f_{i}+g_{i}\right)+\sum_{j \in \mathscr{N}_{i}}\left(a_{i j} V_{j}-\sigma_{i j}\left(\gamma_{j}^{0}-V_{j}\right)\right) \\
-\sum_{j \in \mathscr{N}_{i}} a_{i j} \in \Sigma\left[\bar{x}_{i}\right], \\
\text { and }-\sum_{j \in \mathscr{N}_{i}} a_{i j} \gamma_{j}^{0} \in \Sigma[0], \\
\text { where, } \sigma_{i j} \in \Sigma\left[\bar{x}_{i}\right] \forall j \in \mathscr{N}_{i}, a_{i i} \in \mathbb{R}[0], \\
\text { and } a_{i j} \in \Sigma[0] \forall j \in \mathscr{N}_{i} \backslash\{i\} .
\end{array}
$$

Here $\mathbb{R}[0]$ denotes scalars, $\Sigma[0]$ denotes non-negative scalars and $\bar{x}_{i}$ were defined in (4). If (16) is feasible for each $i \in$ $\{1, \ldots, m\}$, then the origin is exponential stable and the domain $\bigcap_{i=1}^{m} \mathscr{D}_{i}\left[\gamma_{i}^{0}\right]$ is an estimated ROA.

Remark 3 Alternative, and possibly less conservative, formulations of (16) are possible by replacing some of the constraints by an equivalent objective, e.g. replacing (16b) by an objective function $\min \sum_{j \in \mathscr{N}_{i}} a_{i j}$. But those will require centralized computations, e.g. finding eigenvalues of $A$, and are therefore omitted from further discussion.

$\overline{5 a^{1 / p} b^{1 / q}} \leq a / p+b / q$ for $a, b>0, p>1$ and $1 / p+1 / q=1$.

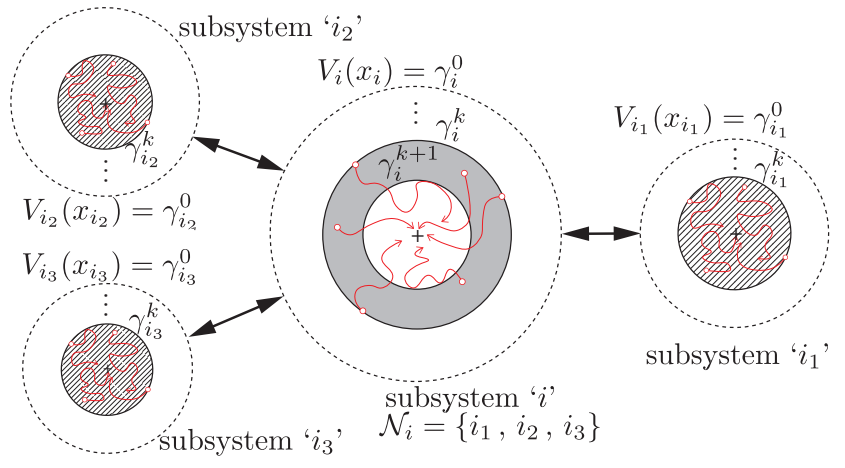

Fig. 1. Distributed coordinated sequential stability certification .

\subsection{Limitations}

The SOS-based single CS approach, proposed in [22], has certain conceptual and computational limitations. Note that larger 'self-decay rates', introduced in (8), favor the CS approach. However, the function $\alpha_{i}(\cdot)$ is generally nonmonotonic, i.e. smaller level-sets need not yield larger selfdecay rates (see Fig. 2(c) in Section 6). Consequently, finding a single set of scalars $a_{i j} \forall i, \forall j \in \mathscr{N}_{i}$, that satisfy the comparison inequalities, including (16b)-(16c), in a large domain $\bigcap_{i=1}^{m} \mathscr{D}_{i}\left[\gamma_{i}^{0}\right]$ could be difficult. Instead, we propose a better approach involving multiple comparison systems (or, multiple CSs). Secondly, the proposed direct approach requires solving subsystem-level SOS problems that involve all the state variables associated with the neighborhoods. Consequently, presence of a large neighborhood can severely affect the overall computational speed, and scalability, of the analysis. A pairwise-interactions based approach can further reduce the computational burden at the subsystems by reducing the size of the SOS problem. Finally, it is not clear how to find the scalars $\gamma_{i}^{0} \forall i$ that define $\bigcap_{i=1}^{m} \mathscr{D}_{i}\left[\gamma_{i}^{0}\right]$, the domain of definition of the CS. It is logical that the set of values for $\gamma_{i}^{0} \forall i$ should be found adaptively, given a disturbance, so that the domain $\bigcap_{i=1}^{m} \mathscr{D}_{i}\left[\gamma_{i}^{0}\right]$ takes a shape that resembles the particular disturbance. In Section 5 we propose a novel framework to circumvent these limitations.

\section{Multiple Comparison Systems}

In this section, we formulate a generalized CSs approach in which we use a sequence of CSs to collectively certify stability under given disturbances. We also propose a framework to parallelize the subsystem-level SOS problems using the pairwise interactions. Fig. 1 illustrates the basic idea behind our proposed formulation. Given any invariant domain $\bigcap_{i=1}^{m} \mathscr{D}_{i}\left[\gamma_{i}^{k}\right], k=0,1,2, \ldots$, the idea is to find the next invariant domain $\bigcap_{i=1}^{m} \mathscr{D}_{i}\left[\gamma_{i}^{k+1}\right]$, with $\mathscr{D}_{i}\left[\gamma_{i}^{k+1}\right] \subseteq \mathscr{D}_{i}\left[\gamma_{i}^{k}\right] \forall i$, such that any trajectory starting from $\bigcap_{i=1}^{m} \mathscr{D}_{i}\left[\gamma_{i}^{k}\right]$ converges exponentially on $\bigcap_{i=1}^{m} \mathscr{D}_{i}\left[\gamma_{i}^{k+1}\right]$. This is done in a distributed way in which each subsystem computes its next invariant levelset and communicates that value to its neighbors, until all the sequences of level-sets converge (to zero, for asymptotic stability). The idea is that, 
Lemma 2 If the subsystem LFs of the interconnected system (3) satisfy the 'multiple CSs' given by,

$$
\forall(k, i): \dot{V}_{i} \leq \sum_{j \in \mathfrak{N}_{i}} a_{i j}^{k}\left(V_{j}-\gamma_{j}^{k+1}\right) \text { on } \bigcap_{j \in \mathscr{N}_{i}} \mathscr{D}_{j}\left[\gamma_{j}^{k}, \gamma_{j}^{k+1}\right]
$$

with $\sum_{j \in \mathscr{N}_{i}} a_{i j}^{k}<0 \forall(k, i)$ and $\sum_{j \in \mathscr{N}_{i}} a_{i j}^{0}\left(\gamma_{j}^{0}-\gamma_{j}^{1}\right)<0 \forall i$, then the system trajectories converge exponentially to $\bigcap_{i=1}^{m} \mathscr{D}_{i}\left[\gamma_{i}^{*}\right]$ where $\gamma_{i}^{*} \forall i$ is the limit of the monotonically decreasing sequence of non-negative scalars $\left\{\gamma_{i}^{k}\right\}, k \in\{0,1,2, \ldots\}$.

Proof Note that the CSs can be written compactly as,

$$
\forall k: \dot{V} \leq A^{k}\left(V-\gamma^{k+1}\right) \text { on } \bigcap_{i=1}^{m} \mathscr{D}_{i}\left[\gamma_{i}^{k}, \gamma_{i}^{k+1}\right],
$$

where $A^{k}=\left[a_{i j}^{k}\right]$ and $\gamma^{k+1}=\left[\begin{array}{llll}\gamma_{1}^{k+1} & \gamma_{2}^{k+1} \ldots \gamma_{m}^{k+1}\end{array}\right]^{T}$. The conditions $\sum_{j \in \mathscr{N}_{i}} a_{i j}^{k}<0 \forall(k, i)$ and $\sum_{j \in \mathscr{N}_{i}} a_{i j}^{0}\left(\gamma_{j}^{0}-\gamma_{j}^{1}\right)<0 \forall i$ imply that $A^{k} \forall k$ is Hurwitz and $\bigcap_{i=1}^{m} \mathscr{D}_{i}\left[\gamma_{i}^{0}\right]$ is invariant. Hence, for each $k$, the system trajectories starting inside $\bigcap_{i=1}^{m} \mathscr{D}_{i}\left[\gamma_{i}^{k}\right]$ converge exponentially to $\bigcap_{i=1}^{m} \mathscr{D}_{i}\left[\gamma_{i}^{k+1}\right]$, while always staying within $\bigcap_{i=1}^{m} \mathscr{D}_{i}\left[\gamma_{i}^{0}\right]$. Note that now the new state variables in the comparison system are $\left(V_{i}-\gamma_{j}^{k+1}\right)$. As the subsystems cross the level sets $\gamma_{i}^{k+1}$, the comparison system changes. Indeed, let's say, the subsystems cross into $\gamma_{i}^{k+1}$ in the order $\{1,2,3, \ldots\}$. After subsystem 1 crosses, the new comparison system is

$$
\begin{aligned}
& \dot{\tilde{V}}_{2: m} \leq A_{2: m}^{k} \tilde{V}_{2: m} \\
& \text { where, } \tilde{V}_{2: m}=\left[\begin{array}{c}
\left(V_{2}-\gamma_{2}^{k+1}\right) \\
\vdots \\
\left(V_{m}-\gamma_{m}^{k+1}\right)
\end{array}\right], A_{2: m}^{k}=\left[\begin{array}{ccc}
a_{22}^{k} & \ldots & a_{2 m}^{k} \\
\vdots & \ddots & \vdots \\
a_{m 2}^{k} & \ldots & a_{m m}^{k}
\end{array}\right] .
\end{aligned}
$$

Note that each matrix in the sequence $A_{2: m}^{k}, \ldots, A_{m: m}^{k}$ remains Hurwitz. Moreover, regardless of the order in which the subsystems cross the level sets $\gamma_{i}^{k+1}$, the sequence of $A$ matrices remains Hurwitz, thus proving finite time convergence to the new level sets.

Corollary $1 \gamma_{i}^{*}=0 \forall i$ implies exponential stability.

Such a formulation, however, is difficult to implement in a distributed algorithm, since the computation of the $i^{\text {th }}$ row of the comparison matrices at the iteration $k$ requires that subsystem- $i$ has knowledge of the $\gamma_{j}^{k+1} \forall j \in \mathscr{N}_{i} \backslash\{i\}$ of its neighbors. A possible approach could be, for each iteration$k$, compute the $k$-th CS iteratively, i.e. using iterations within iterations. But in this work, we restrict ourselves to a simpler formulation, by seeking only diagonal comparison matrices.

Lemma 3 If the subsystem LFs of the system in (3) satisfy

$$
\forall(k, i): \dot{V}_{i} \leq a_{i i}^{k}\left(V_{i}-\gamma_{i}^{k+1}\right) \text { on } \mathscr{D}_{i}\left[\gamma_{i}^{k}, \gamma_{i}^{k+1}\right] \bigcap_{j \in \mathfrak{N}_{i} \backslash\{i\}} \mathscr{D}_{j}\left[\gamma_{j}^{k}\right]
$$

with $a_{i i}^{k}<0 \forall(k, i)$, then the system trajectories converge exponentially to $\bigcap_{i=1}^{m} \mathscr{D}_{i}\left[\gamma_{i}^{*}\right]$ where $\gamma_{i}^{*} \forall i$ is the limit of the monotonically decreasing sequence of non-negative scalars $\left\{\gamma_{i}^{k}\right\}, k \in\{0,1,2, \ldots\}$.

Proof $\bigcap_{i=1}^{m} \mathscr{D}_{i}\left[\gamma_{i}^{k}\right]$ are invariant $\forall k$. The rest is trivial.

Proposition 3 Exponential stability, i.e. $\gamma_{i}^{*}=0 \forall i$, is guaranteed via the multiple (diagonal) CSs approach, if and only if $\nabla V_{i}^{T}\left(f_{i}+g_{i}\right)<0$ on $\mathscr{D}_{i}^{b}\left[\gamma_{i}^{k}\right] \bigcap_{j \in \mathscr{N}_{i} \backslash\{i\}} \mathscr{D}_{j}\left[\gamma_{j}^{k}\right] \forall(k, i)$.

Proof Exponential stability requires $\gamma_{i}^{k+1}<\gamma_{i}^{k} \forall(k, i)$ which immediately proves necessity. The sufficiency follows from the continuity of the polynomial functions.

Corollary 2 Exponential stability is guaranteed if $\alpha\left(\gamma_{i}^{k}\right) \gamma_{i}^{k}>$ $\max _{x \in \mathscr{D}_{i}^{b}\left[\gamma_{i}^{k}\right] \cap_{j \in \mathscr{N}_{i} \backslash\{i\}} \mathscr{D}_{j}\left[\gamma_{j}^{k}\right]} \nabla V_{i}^{T} g_{i} \forall(k, i)$.

The computation of the multiple CSs in Lemma 3 involves two phases. In Phase 1, we search for the level-sets $\gamma_{i}^{0} \in$ $[0,1) \forall i$ such that the system trajectories starting from some initial level-sets $V_{i}\left(x_{i}(0)\right)=v_{i}^{0} \in\left[0, \gamma_{i}^{0}\right] \forall i$, will always stay within $\bigcap_{i=1}^{m} \mathscr{D}_{i}\left[\gamma_{i}^{0}\right]$ which we term as an 'invariant envelope' of $\bigcap_{i=1}^{m} \mathscr{D}_{i}\left[v_{i}^{0}\right]$. In Phase 2, we compute $a_{i i}^{k}$ and $\gamma_{i}^{k+1} \forall(k, i)$.

\subsection{Distributed Construction}

\subsubsection{Phase 1: Find the Invariant Envelope $\bigcap_{i} \mathscr{D}_{i}\left[\gamma_{i}^{0}\right]$}

We search for the smallest $\gamma_{i}^{0} \in\left[v_{i}^{0}, 1\right) \forall i$ that satisfy,

$$
\forall i: \quad \dot{V}_{i} \leq 0 \text { on } \mathscr{D}_{i}^{b}\left[\gamma_{i}^{0}\right] \bigcap_{j \in \mathscr{N}_{i} \backslash\{i\}} \mathscr{D}_{j}\left[\gamma_{j}^{0}\right],
$$

This requires knowledge of the neighbors' expanded levelsets, and hence can only be solved via an iterative process which aims to achieve an agreement between the neighboring subsystems on their individual expanded level-sets. Setting $\hat{\gamma}_{i}^{0}=v_{i}^{0} \forall i$ we compute the monotonically increasing sequences $\left\{\hat{\gamma}_{i}^{l}\right\} \forall i, l \in\{0,1,2, \ldots\}$ satisfying the following,

$$
\begin{gathered}
\forall(l, i): \quad \dot{V}_{i} \leq 0 \text { on } \mathscr{D}_{i}^{b}\left[\hat{\gamma}_{i}^{l+1}\right] \bigcap_{j \in \mathscr{N}_{i} \backslash\{i\}} \mathscr{D}_{j}\left[\hat{\gamma}_{j}^{l}\right], \\
(\mathrm{SOS}):\left\{\begin{array}{c}
-\nabla V_{i}^{T}\left(f_{i}+g_{i}\right)-\sigma_{i i}\left(\hat{\gamma}_{i}^{+1}-V_{i}\right) \\
-\sum_{j \in \mathscr{N}_{i} \backslash\{i\}} \sigma_{i j}\left(\hat{\gamma}_{j}^{l}-V_{j}\right) \in \Sigma\left[\bar{x}_{i}\right], \\
\sigma_{i i} \in \mathbb{R}\left[\bar{x}_{i}\right], \sigma_{i j} \in \Sigma\left[\bar{x}_{i}\right] \forall j \neq i,
\end{array}\right.
\end{gathered}
$$

which is accomplished by finding the smallest $\hat{\gamma}_{i}^{l+1}$, using an incremental-search approach ${ }^{6}$ to handle the bilinear term in $\sigma_{i i}$ and $\hat{\gamma}_{i}^{l+1}$. If $\left\{\hat{\gamma}_{i}^{l}\right\} \forall i$ converge at some $l=L$, we assign $\gamma_{i}^{0} \leftarrow \hat{\gamma}_{i}^{L} \forall i$ and stop.

$\overline{6} \hat{\gamma}_{i}^{+1}$ is increased in small steps until the SOS problem is feasible. 


\subsubsection{Phase 2: Find the Diagonal Comparison Matrices}

With the invariant envelope already found, we can compute the multiple CSs in Lemma 3. At each iteration $k$, each subsystem- $i$ computes $a_{i i}^{k}$ and the smallest $\gamma_{i}^{k+1}$, using a bisection-search on $\gamma_{i}^{k+1}$ over $\left[0, \gamma_{i}^{k}\right]$, which satisfies:

$$
\begin{aligned}
& \forall(k, i): \dot{V}_{i} \leq a_{i i}^{k}\left(V_{i}-\gamma_{i}^{k+1}\right) \text { with } a_{i i}^{k}<0, \\
& \text { everywhere on } \mathscr{D}_{i}\left[\gamma_{i}^{k}, \gamma_{i}^{k+1}\right] \bigcap_{j \in \mathscr{N}_{i} \backslash\{i\}} \mathscr{D}_{j}\left[\gamma_{j}^{k}\right] . \\
&(\mathrm{SOS}):\left\{\begin{array}{r}
-\nabla V_{i}^{T}\left(f_{i}+g_{i}\right)+\left(a_{i i}^{k}-\underline{\sigma}_{i i}\right)\left(V_{i}-\gamma_{i}^{k+1}\right) \\
+\sum_{j \in \mathscr{N}_{i}} \sigma_{i j}\left(V_{j}-\gamma_{j}^{k}\right) \in \Sigma\left[\bar{x}_{i}\right], \\
-a_{i i}^{k} \in \Sigma[0], \underline{\sigma}_{i i} \in \Sigma\left[\bar{x}_{i}\right], \sigma_{i j} \in \Sigma\left[\bar{x}_{i}\right] \forall j \in \mathscr{N}_{i} .
\end{array}\right.
\end{aligned}
$$

We continue until the sequences $\left\{\gamma_{i}^{k}\right\} \forall i$ converge. The exponential stability is guaranteed if $\gamma_{i}^{k+1}=0 \forall i$.

\subsection{Distributed Parallel Construction}

The computational complexities in the SOS problems in Section 5.1 are largely dominated by the dimension of the state-space of the associated neighborhood. To circumvent this limitation, we propose a parallel formulation of the SOS problems based on the pairwise interaction terms. Note that,

Lemma 4 If the subsystem LFs of the system in (3) satisfy

$$
\forall(k, i, j): \nabla V_{i}^{T}\left(w_{i j}^{k} f_{i}+g_{i j}\right) \leq a_{i i, j}^{k}\left(V_{i}-\gamma_{i}^{k+1}\right), w_{i j}^{k} \geq 0
$$

on $\mathscr{D}_{i}\left[\gamma_{i}^{k}, \gamma_{i}^{k+1}\right] \cap \mathscr{D}_{j}\left[\gamma_{j}^{k}\right]$, with $\sum_{j \in \mathscr{N}_{i} \backslash\{i\}} w_{i j}^{k}<1$ and $a_{i i, j}^{k}<$ $0 \forall(k, i)$, then the system trajectories converge exponentially to $\bigcap_{i=1}^{m} \mathscr{D}_{i}\left[\gamma_{i}^{*}\right]$ where $\gamma_{i}^{*} \forall i$ is the limit of the monotonically decreasing sequence of non-negative scalars $\left\{\gamma_{i}^{k}\right\}, k \in$ $\{0,1,2, \ldots\}$.

Proof Note that $\nabla V_{i}^{T}\left(f_{i}+\sum_{j} g_{i j}\right) \leq \sum_{j} \alpha_{i i, j}^{k}\left(V_{i}-\gamma_{i}^{k+1}\right)+(1-$ $\left.\sum_{j} w_{i j}^{k}\right) \nabla V_{i}^{T} f_{i}<\sum_{j} \alpha_{i i, j}^{k}\left(V_{i}-\gamma_{i}^{k+1}\right)$. The rest is trivial.

Note that Lemma 4 can be generalized by replacing the pairwise terms $g_{i j}$ by interaction terms that may involve state variables from two or more neighbors. Next we present an alternative parallelized formulation of the algorithmic steps in Section 5.1 with the help of these 'weights', $w_{i j}^{k}$.

\subsubsection{Phase 1: Find the Invariant Envelope $\bigcap_{i} \mathscr{D}_{i}\left[\gamma_{i}^{0}\right]$}

We set $\hat{\gamma}_{i}^{0}=v_{i}^{0} \forall i$ and compute the sequences $\left\{\hat{\gamma}_{i}^{l}\right\} \forall i$ by finding, for each subsystem- $i$, the smallest $\hat{\gamma}_{i}^{l+1}$ such that $\sum_{j \in \mathscr{N}_{i} \backslash\{i\}} \hat{w}_{i j}^{l}<1$, where the 'weights', $\hat{w}_{i j}^{l}$, are defined as,

$$
\begin{aligned}
& \forall(l, i, j): \hat{w}_{i j}^{l}=\min \left\{\begin{array}{l|l}
\hat{w} & \begin{array}{c}
\nabla V_{i}^{T}\left(\hat{w} f_{i}+g_{i j}\right) \leq 0 \\
\text { on } \mathscr{D}_{i}^{b}\left[\hat{\gamma}_{i}^{l+1}\right] \cap \mathscr{D}_{j}\left[\hat{\gamma}_{j}^{l}\right]
\end{array}
\end{array}\right\}, \\
& (\mathrm{SOS}):\left\{\begin{array}{c}
\underset{\sigma_{i i}, \sigma_{i j}}{\operatorname{minimize}} \hat{w}, \text { subject to: } \\
-\nabla V_{i}^{T}\left(\hat{w} f_{i}+g_{i j}\right)-\sigma_{i i}\left(\hat{\gamma}_{i}^{l+1}-V_{i}\right) \\
\quad-\sigma_{i j}\left(\hat{\gamma}_{j}^{l}-V_{j}\right) \in \Sigma\left[x_{i}, x_{j}\right], \\
\sigma_{i i} \in \mathbb{R}\left[x_{i}, x_{j}\right], \sigma_{i j} \in \Sigma\left[x_{i}, x_{j}\right] .
\end{array}\right.
\end{aligned}
$$

This is done using an incremental-search approach on $\hat{\gamma}_{i}^{l+1}$. If $\left\{\hat{\gamma}_{i}^{l}\right\} \forall i$ converge at $l=L$, we assign $\gamma_{i}^{0} \leftarrow \hat{\gamma}_{i}^{L} \forall i$ and stop.

\subsubsection{Phase 2: Find the Diagonal Comparison Matrices}

Phase 2 of the process essentially remains same as the one in described in Section 5.1.2 except that we need to additionally compute the weights $w_{i j}^{k}$. For each subsystem- $i$, we perform a bisection-search on the smallest $\gamma_{i}^{k+1}$ over $\left[0, \gamma_{i}^{k}\right]$, such that $\sum_{j \in \mathscr{N}_{i}} w_{i j}^{k}<1$, where the weights $w_{i j}^{k}$ are defined as,

$$
\begin{aligned}
& \forall(k, i, j): w_{i j}^{k}:= \\
& \min \left\{\begin{array}{c|c}
w & \begin{array}{c}
\nabla V_{i}^{T}\left(w f_{i}+g_{i j}\right) \leq a_{i i, j}^{k}\left(V_{i}-\gamma_{i}^{k+1}\right) \\
\text { on } \mathscr{D}_{i}\left[\gamma_{i}^{k}, \gamma_{i}^{k+1}\right] \cap \mathscr{D}_{j}\left[\gamma_{j}^{k}\right], a_{i i, j}^{k}<0 .
\end{array}
\end{array}\right\}
\end{aligned}
$$

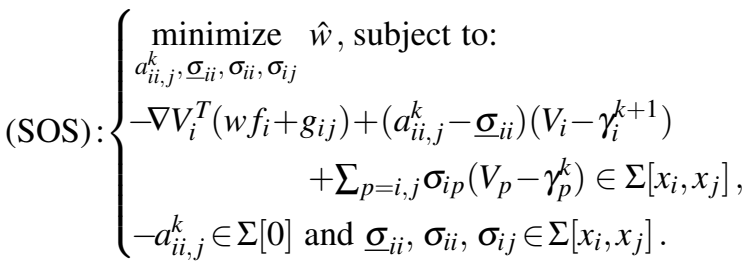

At each $k$, we solve the above bisection search to find the smallest $\gamma_{i}^{k+1} \forall i$ satisfying $\sum_{j \in \mathscr{N}_{i}} w_{i j}^{k}<1$, until $\left\{\gamma_{i}^{k}\right\} \forall i$ converges. If $\gamma_{i}^{k+1}=0 \forall i$, the exponential stability is guaranteed.

\section{Numerical Example}

We consider a network of nine modified Van der Pol 'oscillators' [38], with parameters of each oscillator chosen to make them individually stable. Each Van der Pol is treated as an individual subsystem, with the following interconnections,

$$
\begin{aligned}
& \mathscr{N}_{1}:\{1,2,5,9\} \quad \mathscr{N}_{2}:\{2,1,3\} \mathscr{N}_{3}:\{3,2,8\} \\
& \mathscr{N}_{4}:\{4,6,7\} \quad \mathscr{N}_{5}:\{5,1,6\} \mathscr{N}_{6}:\{6,4,5\} \\
& \mathscr{N}_{7}:\{7,4,8,9\} \quad \mathscr{N}_{8}:\{8,3,7\} \mathscr{N}_{9}:\{9,1,7\} .
\end{aligned}
$$

Each subsystem $i \in\{1,2, \ldots, 9\}$ has two state variables, $x_{i}=$ $\left[\begin{array}{ll}x_{i, 1} & x_{i, 2}\end{array}\right]^{T}$. After shifting the equilibrium point to the origin 


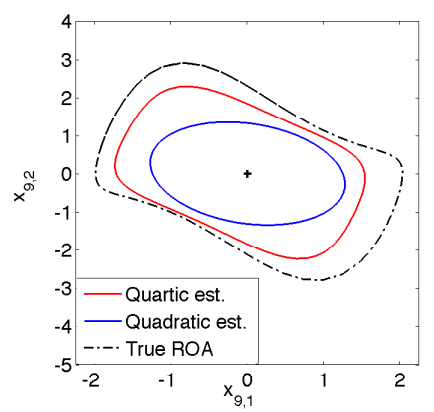

(a) ROAs for isolated subsystem 9

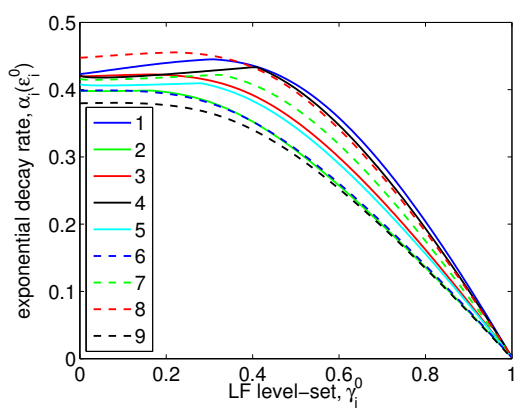

(b) 'Self-decay' rates for quadratic LFs

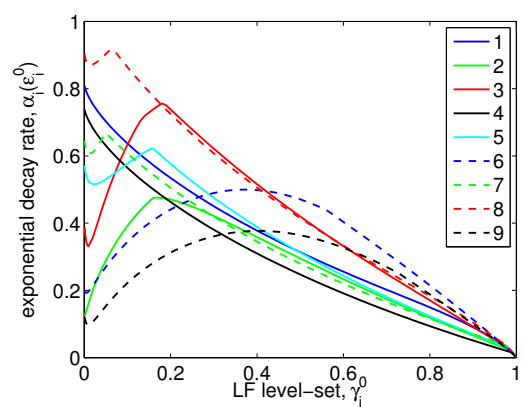

(c) 'Self-decay' rates for quartic LFs

Fig. 2. Characteristics of subsystem LFs: (a) comparison of estimated ROAs, (b)-(c) exponential 'self-decay' rates.

(see Appendix A for details), the subsystem dynamics in the presence of the neighbor interactions are given by

$$
\begin{aligned}
& f_{i}\left(x_{i}\right)=\left[x_{i, 2}, \mu_{i} x_{i, 2}\left(c_{i}^{(1)}-c_{i}^{(2)} x_{i, 1}-x_{i, 1}^{2}\right)-c_{i}^{(3)} x_{i, 1}\right]^{T} \\
& g_{i j}\left(x_{i}, x_{j}\right)=\left[0, \beta_{i j}^{(1)} x_{j, 2}+\beta_{i j}^{(2)} x_{j, 2} x_{i, 1}\right]
\end{aligned}
$$

where, $c_{i}^{(1)}=1-\left(0.5 c_{i}^{(2)}\right)^{2}, c_{i}^{(3)}=1-\sum_{j \in \mathscr{N}_{i}\{i\}}\left(0.5 \beta_{i j}^{(2)} c_{i}^{(2)}-\right.$ $\left.\beta_{i j}^{(1)}\right), \mu_{i}, \beta_{i j}^{(1)}$ and $\beta_{i j}^{(2)}$ are chosen randomly and $c_{i}^{(2)}$ are related to the equilibrium point before shifting. Polynomial LFs for the isolated (no interaction) subsystems are computed using the expanding interior algorithm (Section 3 ). Fig. 2(a) shows that a quartic LF estimates the 'true' ROA of the isolated subsystems (obtained via time-reversed simulation) better than a quadratic LF. However, a better estimate of the isolated ROAs does not necessarily translate into better stability certificates for the interconnected system, as illustrated later. The 'self-decay rates' are plotted in Figs. 2(b)-2(c) for a range of level-sets from 0 to 1. For each subsystem, as $\gamma_{i}^{0}$ approaches $1, \alpha_{i}\left(\gamma_{i}^{0}\right)$ approaches 0 . Thus it is impossible to obtain a Hurwitz comparison matrix when the initial conditions lie close to the boundary of the estimated ROAs. Moreover, note that the evolution of the self-decay rates is generally non-monotonic. An attempt to find a single CS valid all the way to the origin is generally difficult, since the row-sum values of the single comparison matrix will be limited by the lowest self-decay rate. A multiple CS approach, however, can still guarantee exponential convergence to some level-sets close to the origin.

In Fig. 3, we compare the traditional and the direct approaches of computing a CS (using the quadratic LFs). Choosing $\gamma_{1}^{0}=\gamma_{2}^{0}=\ldots=\gamma_{9}^{0}$, and varying their values, we compute the comparison matrices in (15) using SOS-based direct approach in (16), by replacing the constraint (16b) by an objective of minimizing $\sum_{j} a_{i j} \forall i$. Also, we find the comparison matrices using the traditional approach, from (13) and (12). The maximum of the real parts of the eigenvalues (denoted by $\operatorname{Re}(\lambda)$ ) and the maximum row-sum of the comparison matrices are plotted, for varying level-sets. Clearly, the SOS-based direct method yields improved stability cer-

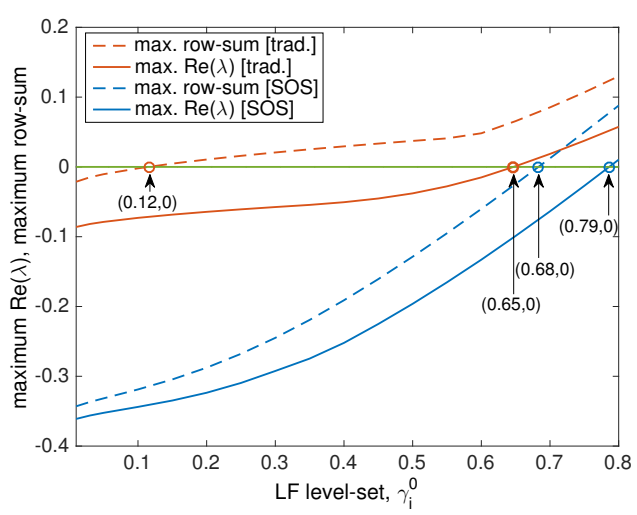

Fig. 3. Stability properties of the single comparison matrices obtained via traditional and direct methods (using quadratic LFs)

tificates. Further note that the maximal (uniform) level-set for which both the maximum row-sum value and the maximum of $\operatorname{Re}(\lambda)$ are negative gives an estimate of the ROA of the full interconnected system. Thus $\bigcap_{i=1}^{9} \mathscr{D}[0.683]$ is an estimate of the ROA. Next we compare the performances of the single CS approach and the multiple CSs approach (with and without the parallel computation), in Fig. 4. For each subsystem- $i$, we plot the maximal (uniform) level-set for which either the row-sum is negative (single CS), or a strict convergence is achieved at iteration-0, i.e. $\gamma_{i}^{1}<\gamma_{i}^{0}$ (multiple CS). When not considering the parallel implementation, the multiple CS approach outperforms the single CS in estimating the invariance (since we focus only at iteration-0). However, the parallel implementation, while achieving computational tractability for larger systems, yields more conservative certificates.

Next we use an example to illustrate a couple of key observations. Fig. 5 shows the stability analysis results on an arbitrarily generated initial condition (or disturbance) using both quadratic and quartic LFs, and multiple CSs. Note in Fig. 5(b) that the initial level-set lie outside the estimated ROA obtained in Fig. 3. By allowing the analysis to be dependent on the particular initial condition, we are able to find a suitably shaped stability region. Further note that, while the quadratic LFs-based multiple CSs analysis certifies ex- 


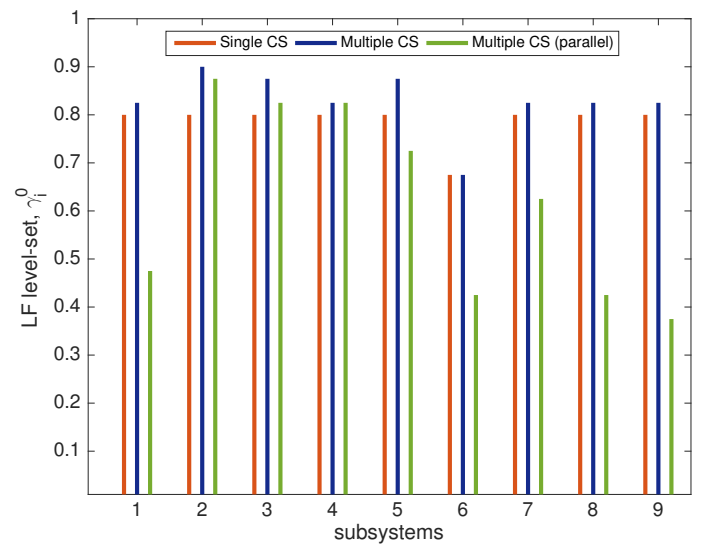

Fig. 4. Comparison between three approaches - single CS; distributed multiple CSs; and distributed parallel multiple CSs.

ponential stability (in Fig. 5(b)), the quartic LFs can only guarantee exponential convergence to a domain $\bigcap_{i=1}^{9} \mathscr{D}_{i}\left[\gamma_{i}^{*}\right]$ very close to the origin, with $\gamma_{2}^{*}=0.023$ and $\gamma_{3}^{*}=0.016$ (in Fig. 5(c)). In fact, this domain of convergence is characteristic of the system and the (quartic) LFs used, and is independent of the initial condition. Referring to Fig. 2(c), the low self-decay rates of the quartic LFs for subsystems 2 and 3 explain the convergence away from the origin (Corollary 2). Thus, while the quartic LFs may yield better estimates of the isolated ROAs (Fig. 2(a)) compared to the quadratic LFs, and hence are able to analyze a larger number of initial conditions, they fail to certify exponential stability. This suggests that it may be useful to switch from quartic LFs to the quadratic LFs as the $\gamma_{i}^{k} \forall(k, i)$ decrease. We also noted that, in the quartic LF-based analysis subsystems-6 and 7 underwent an expansion (Phase 1) from $\left(v_{6}^{0}, v_{7}^{0}\right)=(0.015,0.002)$ to $\left(\gamma_{6}^{0}, \gamma_{7}^{0}\right)=(0.151,0.013)$, while none of the subsystems underwent expansion using quadratic LF-based analysis.

Remark 4 Note that the algorithm estimates the exponential convergence rates rather conservatively. While this issue may be resolved by constraining the row-sum values to be less than some chosen negative number, it will likely delay the convergence of the stability algorithm.

\section{Conclusion}

We have used vector Lyapunov functions to design an iterative, distributed and parallel algorithm to certify exponential stability of a nonlinear network, under initial disturbances. The algorithm requires one-time computation of Lyapunov functions of the isolated subsystems, and minimal real-time communications between the neighboring subsystems. It is shown that the proposed SOS-based direct approach towards computation of the single comparison system leads to less conservative certificates than the traditional methods. Further, a generalization has been proposed via multiple comparison systems, which has been found to yield improved results compared to the single comparison system approach.
Using the pairwise interactions, a parallel implementation is also proposed, which enables the algorithm to scale up smoothly with the size of the largest neighborhood. The distributed stability analysis algorithm has been tested on an arbitrary network of nine Van der Pol systems, using vectors of quadratic and quartic Lyapunov functions. It is easy to visualize a multi-agent distributed coordinated control framework where each subsystem ('agent') will coordinate with its neighbors to design local control policies to stabilize the system under disturbances. Finally, it would be interesting to explore the applicability of the proposed algorithm on real-world problems, such as the transient stability analysis of large-scale structure-preserving power system networks.

\section{References}

[1] Ahmadi, A. A. and A. Majumdar. DSOS and SDSOS optimization: LP and SOCP-based alternatives to sum of squares optimization. In Information Sciences and Systems (CISS), 2014 48th Annual Conference on, pages 1-5, Mar 2014.

[2] J. Anderson, Y.-C. Chang, and A. Papachristodoulou. Model decomposition and reduction tools for large-scale networks in systems biology. Automatica, 47(6):1165-1174, 2011.

[3] M. Anghel, F. Milano, and A. Papachristodoulou. Algorithmic construction of Lyapunov functions for power system stability analysis. Circuits and Systems I: Regular Papers, IEEE Transactions on, 60(9):2533-2546, Sep 2013.

[4] M. Araki. Stability of large-scale nonlinear systems quadraticorder theory of composite-system method using M-matrices. IEEE Transactions on Automatic Control, 23(2):129 - 142, 1978.

[5] F. N. Bailey. The application of Lyapunov's second method to interconnected systems. J. SIAM Control, 3:443 - 462, 1966.

[6] E. F. Beckenbach and R. Bellman. Inequalities. Spring-Verlag, New York/Berlin, 1961.

[7] H. E. Bell. Gershgorin's theorem and the zeros of polynomials. American Mathematical Monthly, pages 292-295, 1965.

[8] R. Bellman. Vector Lyapunov functions. SIAM Journal of Control, 1(1):32-34, 1962.

[9] F. Brauer. Global behavior of solutions of ordinary differential equations. Journal of Mathematical Analysis and Applications, 2(1):145-158, 1961.

[10] G. Chesi. Estimating the domain of attraction for non-polynomial systems via LMI optimizations. Automatica, 45:1536-1541, 2009.

[11] G. Chesi. LMI techniques for optimization over polynomials in control: a survey. IEEE Transactions on Automatic Control, 55(11):2500 - 2510, 2010.

[12] G. Chesi. Domain of Attraction: Analysis and Control via SOS programming. Lecture Notes in Control and Information Sciences. Springer, London, 2011.

[13] R. Conti. Sulla prolungabilità delle soluzioni di un sistema di equazioni differenziali ordinarie. Bollettino dell'Unione Matematica Italiana, 11(4):510-514, 1956.

[14] Šiljak D. D. Decentralized Control of Complex Systems. Mathematics in Science \& Engineering. Academic Press, Boston, 1991.

[15] R. Genesio, M. Tartaglia, and A. Vicino. On the estimation of asymptotic stability regions: State of the art and new proposals. IEEE Transactions on Automatic Control, 30(8):747-755, Aug. 1985.

[16] S. A. Gershgorin. Uber die abgrenzung der eigenwerte einer matrix. Izv. Akad. Nauk. USSR Otd. Fiz.-Mat. Nauk, 1(6):749-754, 1931. 


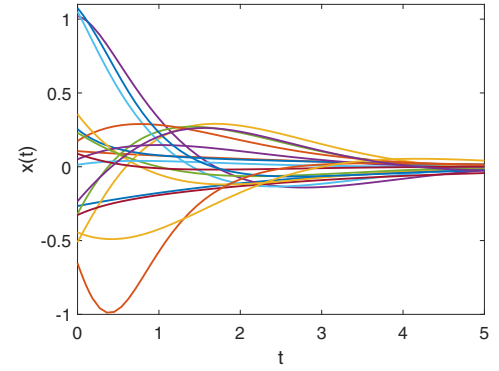

(a) System states under disturbance

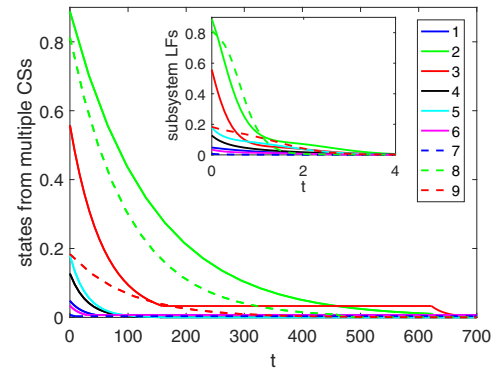

(b) Quadratic LFs analysis

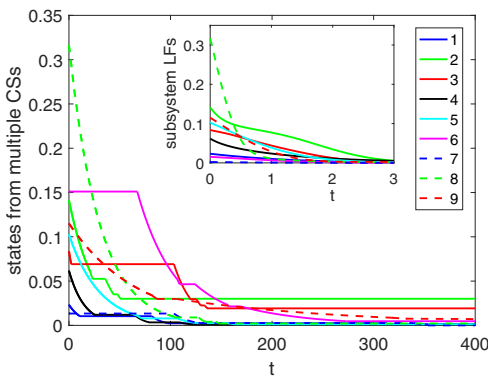

(c) Quartic LFs analysis

Fig. 5. Analysis of (a) a given stable initial condition using (b) quadratic and (c) quartic LFs, and multiple CSs.

[17] W. M. Haddad and V. Chellaboina. Nonlinear Dynamical Systems and Control. Princeton University Press, New Jersey, 2008.

[18] Z. W. Jarvis-Wloszek. Lyapunov Based Analysis and Controller Synthesis for Polynomial Systems using Sum-of-Squares Optimization. $\mathrm{PhD}$ thesis, University of California, Berkeley, CA, 2003.

[19] L. Jocic, M. Ribbens-Pavella, and D.D. Šiljak. Multimachine power systems: Stability, decomposition, and aggregation. Automatic Control, IEEE Transactions on, 23(2):325-332, Apr 1978.

[20] I. Karafyllis and M. Papageorgiou. Global exponential stability for discrete-time networks with applications to traffic networks. IEEE Transactions on Control of Network Systems, 2(1):68-77, 2015

[21] H. K Khalil. Nonlinear Systems. Prentice Hall, New Jersey, 1996.

[22] S. Kundu and M. Anghel. Computation of linear comparison equations for stability analysis of interconnected systems. In 54th IEEE Conf. on Decision and Control, pages 1762-1768, Dec 2015.

[23] S. Kundu and M. Anghel. Stability and control of power systems using vector Lyapunov functions and sum-of-squares methods. In European Control Conference, pages 253-259, July 2015.

[24] S. Kundu and M. Anghel. A sum-of-squares approach to the stability and control of interconnected systems using vector Lyapunov functions. In The 2015 American Control Conference, pages 5022 5028, July 2015.

[25] J.-B. Lasserre. Moments, Positive Polynomials and Their Applications, volume 1. World Scientific, 2009.

[26] A. M. Lyapunov. The General Problem of the Stability of Motion. Kharkov Math. Soc., Kharkov, Russia, 1892.

[27] Mason, R. P. and A. Papachristodoulou. Chordal sparsity, decomposing SDPs and the Lyapunov equation. In American Control Conference (ACC), 2014, pages 531-537, Jun 2014.

[28] N Michel, A. On the status of stability of interconnected systems. IEEE Transactions on Automatic Control, 28(6):639-653, 1983.

[29] A. Papachristodoulou, J. Anderson, G. Valmorbida, S. Prajna, P. Seiler, and P. A. Parrilo. SOSTOOLS: Sum of squares optimization toolbox for MATLAB. 2013. Available from http://www.eng.ox.ac.uk/control/sostools.

[30] A. Papachristodoulou and S. Prajna. Positive Polynomials in Control, chapter Analysis of non-polynomial systems using the sum of squares decomposition, pages 23-43. Springer-Verlag, 2005.

[31] P. A. Parrilo. Structured Semidefinite Programs and Semialgebraic Geometry Methods in Robustness and Optimization. $\mathrm{PhD}$ thesis, Caltech, Pasadena, CA, 2000.

[32] S. Prajna, A. Papachristodoulou, P. Seiler, and P. A. Parrilo, Positive Polynomials in Control, chapter SOSTOOLS and Its Control Applications, pages 273-292. Springer-Verlag, 2005.

[33] M. Putinar. Positive polynomials on compact semi-algebraic sets. Indiana University Mathematics Journal, 42(3):969-984, 1993.

[34] J. F. Sturm. Using SeDuMi 1.02, a MATLAB toolbox for optimization over symmetric cones. Optimization Methods and
Software, 11-12:625-653, Dec. 1999. Software available at http://fewcal.kub.nl/sturm/software/sedumi.html.

[35] W. Tan. Nonlinear Control Analysis and Synthesis using Sumof-Squares Programming. PhD thesis, University of California, Berkeley, CA, 2006.

[36] M. Thoma and M. Morari, editors. Positive Polynomials in Control, volume 312 of Lecture Notes in Control and Information Sciences. Springer-Verlag, 2005.

[37] B. Tibken. Estimation of the domain of attraction for polynomial systems via LMIs. In Proceedings of the $39^{\text {th }}$ IEEE Conference on Decision and Control, volume 4, pages 3860 - 3864, 2000.

[38] B. Van der Pol. On relaxation-oscillations. The London, Edinburgh, and Dublin Philosophical Magazine and Journal of Science, 2(11):978-992, 1926

[39] D. D. Šiljak. Stability of large-scale systems under structural perturbations. Systems, Man and Cybernetics, IEEE Transactions on, SMC-2(5):657-663, Nov 1972

[40] D. D. Šiljak. Large Scale Dynamic Systems: Stability and Structure. System Science and Engineering. North-Holland, New York, 1978.

[41] S. Weissenberger. Stability regions of large-scale systems. Automatica, 9(6):653-663, 1973.

[42] Z. J. Wloszek, R. Feeley, W. Tan, K. Sun, and Andrew Packard. Positive Polynomials in Control, chapter Control Applications of Sum of Squares Programming, pages 3-22. Springer-Verlag, Berlin, Heidelberg, 2005.

[43] D. Xu, X. Wang, Y. Hong, Z. P. Jiang, and S. Xu. Output Feedback Stabilization and Estimation of the Region of Attraction for Nonlinear Systems: A Vector Control Lyapunov Function Perspective. IEEE Transactions on Automatic Control, PP(99):1-1, 2016.

\section{A Model Description}

Subsystem dynamics in the original state variables (i.e. before shifting), $\tilde{x}_{i}=\left[\begin{array}{ll}\tilde{x}_{i, 1} & \tilde{x}_{i, 2}\end{array}\right]^{T}$, are given by $\dot{x}_{i}=\tilde{f}_{i}\left(\tilde{x}_{i}\right)+$ $\sum_{j \in \mathscr{N}_{i} \backslash\{i\}} \tilde{g}_{i j}\left(\tilde{x}_{i}, \tilde{x}_{j}\right) \forall i$, where $\tilde{f}_{i}\left(\tilde{x}_{i}\right)=\left[\tilde{x}_{i, 2}, \mu_{i} \tilde{x}_{i, 2}\left(1-\tilde{x}_{i, 1}^{2}\right)-\right.$ $\left.\tilde{x}_{i, 1}\right]^{T}$ and $\tilde{g}_{i j}\left(\tilde{x}_{i}, \tilde{x}_{j}\right)=c_{i j}+\tilde{\beta}_{i j}^{(1)}\left(x_{i, 1}-x_{j, 2}\right)+\beta_{i j}^{(2)} \tilde{x}_{j, 2} \tilde{x}_{i, 1}$. $\mu_{i} \in[-3,-1], c_{i j} \in[-0.2,0.2], \tilde{\beta}_{i j}^{(1)} \in[-0.1,0.1]$ and $\beta_{i j}^{(2)} \in$ $[-0.1,0.1]$ are chosen randomly. By shifting the equilibrium point $\tilde{x}_{i}^{*}=\left\{\sum_{j} c_{i j} /\left(1-\sum_{j} \tilde{\beta}_{i j}^{(1)}\right), 0\right\} \forall i$, to origin with $x_{i}=$ $\tilde{x}_{i}-\tilde{x}_{i}^{*} \forall i$, we obtain (23), where $c_{i}^{(2)}=2 \sum_{j} c_{i j} /\left(1-\sum_{j} \tilde{\beta}_{i j}^{(1)}\right)$, and $\beta_{i j}^{(1)}=0.5 \beta_{i j}^{(2)} c_{i}^{(2)}-\tilde{\beta}_{i j}^{(1)}$. 